\title{
Survey on Recommendation of E-commerce products to existing and new user by Analyzing Social data along with e-commerce data
}

\author{
P P Nagarale ${ }^{1}$ | Dr. K B Manwade ${ }^{2}$
}

\begin{abstract}
${ }_{1}^{1}$ Department of Computer Science and Engineering, Ashokrao Mane Group of Institutions, Vathar, Maharashtra, India. ${ }^{2}$ Department of Computer Science and Engineering, Sanjeevan Engineering and Technology Institute, Panhala, Maharashtra, India.
\end{abstract}

To Cite this Article

P P Nagarale and Dr. K B Manwade, "Survey on Recommendation of E-commerce products to existing and new user by Analyzing Social data along with e-commerce data", International Journal for Modern Trends in Science and Technology, Vol. 06, Issue 07, July 2020, pp.:78-84; https://doi.org/10.46501/IJMTST060712

Article Info

Received on 16-June-2020, Revised on 21-June-2020, Accepted on 28-June-2020, Published on 07-July-2020.

\section{ABSTRACT}

At this moment, the line between Online Media and E-exchange is diminishing. Various online business destinations support the arrangement of social login where customers can sign on the locales using their relational association characters, for instance, their Face book or Twitter accounts. Customers can moreover post their as of late purchased things on smaller scale web journals with associations with the online business thing webpage pages. In any case, customers won't hit a thing in case they can't see it, or they are not roused by the thing the suggesting system expects a fundamental activity in discovering interest things from close unending stock and demonstrating them to likely customers. Be that as it may, two issues are destroying the suggesting structure. One is "the way to manage new customers", and the other is "the methods by which to amaze customers." They can share their purchase nuances through online systems administration media using the association of that purchased thing from that electronic business page. Focusing on the thing proposition to the customers on e-trade destinations by methods for using the information or capacity got from the customers' social records. In cold-start conditions, this will enable us to review the prerequisites of the customer. Cold-Start is a state when the client signs in to the online business site considering the way that and don't have any data about the past occasions, of buys, shopping structures, etc as it isn't yet made or open. They can use the customer's social record information from the customer's posts, friends, share, etc for their bit of leeway. They will apply information mining estimations to get to the little extension that makes the client has made and empty the steady catchphrases and in this way, this information from the tinier degree online journals changes into the clarification behind thing recommendation in Chile beginning conditions. The two segments are uninhibited revealed in the planning sort out and moved into the last proposal for new customers. Moreover, reasonably detail the two issues into a bound together objective and present iterative development estimation. The brisk improvement of the proposed framework is to diminish the multifaceted nature and broad speculative appraisals there are given to check the impediment of our strategy.

KEYWORDS: Cold-star recommendation long-tail recommendation, transfer learning, E-commerce.

Copyright (C) 2014-2020 International Journal for Modern Trends in Science and Technology

DOI: https://doi.org/10.46501/IJMTST060712

78 International Journal for Modern Trends in Science and Technology 


\section{INTRODUCTION}

In concrete, proposed learning the two customers and thing incorporates depiction (called utilizer presenting and thing installing, solely) from data amassed from web business webpage using dreary neural structures and a brief timeframe later apply a decent propensity boosting trees framework to change customer's informal communication highlights into utilizer embedding's. By then build up a portion predicated structure factorization approach that can use the wise utilizer installing for cold-start thing recommendations. The dataset from the most enormously mammoth Chinese micro blogs accommodation SINA WEIBO2 (social networking and micro blogging service based in China) and the most infinitely colossal Chinese B2C (Business-to-consumer) online business web page, containing an aggregate of 20,638 associated customers. There is no quick information open for the crisp starting issue to get from the side of data [1]. In particular, sway the social information of customer's amigo parties, unequivocally vested get-togethers, and page love as side data in our assessments. From the beginning, mapping limits which guide side data to client thing relationship are found on the arranging dataset. By that point, move the educated information to new customers. Given a course of action of the close huge stock, notable things are only a little division of the full-scale things. These things, in any case, have a positive relationship with a larger piece of customers. In a customer thing structure, the standard things can be revealed by a low-position work. Simultaneously, long-tail things are a tremendous bit of the stock; in any case, they have a positive relationship with just a ton of clients. In this way, their relationship can be tended to by a small breaking point. In that capacity, pass on two fundamental parts, a low-position part, and conflicting part, in detail to oversee both the central segments and the distinguishing strength factors, freely. To portray a system that maps such substance (for instance customer or thing) credits to the latest features of a matrix (or higher dimensional) factorization model with such mappings, the components of an MF model arranged by standard strategies can be applied to the new customer and the new thing issue while holding its focal points, explicitly, speed and insightful precision [2].

This examination gives a study of various arrangements proposed to address the new client cold-start issue in suggestion frameworks. The rest of the paper is organized as follows:

The cold-start, the issue is generally perceived and appropriately explored inconvenience for recommender frameworks. Recommender frameworks structure a particular kind of Information separating (IF) strategy that endeavors to offer insights objects (online business, motion pictures, track, books, data, depictions, site pages) that are plausible important to the client [3]. By and large, a recommender framework looks at the client's profile to some reference attributes. those characteristics can be identified with object qualities (content-based separating) or the client's social condition and past conduct (community-oriented sifting) contingent upon the framework, the client might be identified with different sorts of collaborations: appraisals, bookmarks, buys, likes, amount of page visits [4].

There are two different forms of the cold-start problem in RS (Recommender systems):

(1) New user cold-start problem

(2) New item cold-start problem.

In the new client cold-start issue, another client is acquainted with the framework, and RS (Recommender System) faces issues in giving suggestions as it has no data about the client [5]. In the new thing cold-start issue, the framework has no appraisals for the new thing and it faces trouble in deciding an objective client for the thing. Out of the two sorts of cold beginning issues, the new client cold-start issue is progressively troublesome [6].

\section{RECOMMENDATION SYSTEM}

This work relies upon a proposed structure, and it is a subpart of the information isolating system that attempts to envision the "rating" or "tendency" a customer would accommodate a thing recommender structure incorporates a class of procedures and checks which can propose "significant" things to clients. In a perfect world, the proposed things are as proper to the client as would be secured, so the client can pull in with those things: YouTube accounts, reports, online things, etc. [7]. 
Items are positioned by their pertinence, and the most significant ones have appeared to the client. The significance is something that the recommender framework must decide and is principally founded on recorded information. On the off chance that you've as of late viewed YouTube recordings about elephants, at that point, YouTube is going to begin indicating you a ton of elephant recordings with comparative titles and topics.

Recommender systems are generally divided into two main categories: 1. Collaborative filtering 2.Content-based systems [8].

\section{CLASSIFICATION OF RECOMMENDATION SYSTEMS}

This segment presents a characterization of suggestion frameworks in the E-framework considering a few components. To begin with, Subsection surveys the pre-handling procedures, highlights, and models utilized so as to speak to the indexed lists. Next, Subsection presents the most famous bunching calculations utilized to aggregate the website pages identified with every person with a similar name. Following that, the principal part of the proposed framework that considers the really suggested joins which appear therefore individual an inquiry [9].

The period of proposal frameworks in the E-system comprises representing the list of items so as to treat them consequently in the right way. This stage is generally made out of the accompanying advances:

\section{Pre-processing:}

In this step, the output is processed from their original format by means of several techniques.

Feature selection:

The primary objective of this progression is to choose appropriate highlights to separate various people with a similar name accurately.

\section{Representation model:}

In this progression, the list items are spoken to by methods for certainly suggested joins. From one perspective, these models dole out an incentive to each component speaking to its significance as for the website page it has a place with, or to all the indexed lists recovered by the internet searcher. Then again, these models permit the correlation of pages by methods for conduct activities.

\begin{tabular}{|l|l|l|l|l|}
\hline Learning Method & Loss Function & $\begin{array}{l}\text { Number of } \\
\text { clusters: } \\
\text { Predetermined of } \\
\text { Data dependent }\end{array}$ & $\begin{array}{l}\text { Cluster shape: } \\
\text { isotropic or } \\
\text { anisotropic? }\end{array}$ & $\begin{array}{l}\text { Parameter } \\
\text { Estimation } \\
\text { Algorithm }\end{array}$ \\
\hline K-means & $\begin{array}{l}\text { Within-class } \\
\text { squared distance } \\
\text { from mean }\end{array}$ & Predetermined & Isotropic & K-means \\
\hline $\begin{array}{l}\text { Gaussian Mixture } \\
\text { Models(identity } \\
\text { covariance) }\end{array}$ & $\begin{array}{l}\text {-log P(X), } \\
\text { (equivalent to } \\
\text { within-class } \\
\text { squared distance } \\
\text { from mean) }\end{array}$ & Predetermined & Isotropic & Expectation \\
Maximization \\
(EM)
\end{tabular}

Table 1: Comparison of Clustering Algorithm

\section{LITERATURE REVIEW}

This is work [1] proposed a novel response for cross-site page cold-start thing recommendations, which means to endorse things from online business locales to customers at the individual to individual correspondence goals are cold starting conditions, trouble that has now and again been explored beforehand. A huge test is a way to utilize data removed from long range casual correspondence goals for cross-site cold-start thing proposals. This paper proposed to use associated customers over the individual to individual correspondence goals and online business destinations (customers who have relational cooperation records and Purchases on electronic business locales). As an expansion to depict long-extend relational correspondence features to another segment depiction for thing recommendation.

In unequivocal, it proposed learning the two customers and things incorporate depictions (considered customer embedding's and thing embedding's, independently) from data assembled from electronic business locales using discontinuous neural frameworks and a while later 
applies a modified tendency boosting tree procedure to trans-structure customers' long-ago relational correspondence features into customer embedding's' Then developed a component-based lattice factorization approach which can utilize the academic customer introducing's for cold-start thing recommendation. Preliminary outcomes on a colossal dataset worked from the greatest Chinese micro blogging organization SINA WEIBO and the greatest Chinese B2C E-business webpage JINGDONG has exhibited the practicality of this structure.

The accessibility of the sheer volume of online item [2] audits makes it conceivable to infer certain segment data of item adopters from the survey reports. This paper proposed a novel way to deal with the extraction of item adopters specifies from online audits. The extricated item adopters are then arranged into some unique segment client gatherings. The amassed segment data of numerous item adopters can be utilized to portray the two items and clients, which can be joined into a suggested technique utilizing weighted regularized network factorization. These exploratory outcomes on more than 15 million surveys slithered from JINGDONG, the biggest B2C internet business site in China, show the achievability and adequacy of this proposed structure for item suggestion.

As an enormous number of versatile (applications) [3] are promptly accessible, clients experience issues in distinguishing applications that are pertinent to their inclinations. Suggested frameworks that rely upon past client evaluations (i.e., shared sifting, or $\mathrm{CF}$ ) can address this issue for applications that have adequate appraisals from past clients. In any case, for applications that are recently discharged, CF doesn't have any client appraisals to put together proposals with respect to, which prompts the Cold-Start issue. In this paper, depicted a technique that represents early data called from Twitter to give important proposals in such chilly beginning circumstances. Here use Twitter handles to get to an application's Twitter record and concentrate the IDs of their Twitter-followers. This makes pseudo-chronicles that contain the IDs of Twitter customers motivated by an application and subsequently apply an inactive segment to make inert social affairs. At test time, a target customer searching for recommendations is mapped to these inactive social affairs. By using the transitive relationship of dormant get-togethers to applications, these assessments check the probability of the customer appreciating the application. It shows that by joining information from Twitter, this strategy overcomes the difficulty of Cold-Start application proposition and by and large beats other bleeding-edge recommendation systems by up to $33 \%$.

The two errands of KDDCup 2012 [4] are anticipating the adherents of a micro-blogger (track 1) and to foresee the active visitor clicking percentage of the advertisements (track 2). From the outset, the two errands appear to be unique, be that as it may, they share two significant difficulties. In the first place, the primary factors in both issue settings are of the enormous straight out area. Assessing variable collaborations between these sorts of factors is troublesome with standard AI models and factorization models have gotten well known for such information. Besides, numerous extra indicator factors are accessible which requires adaptable models dependent on highlight building to encourage the model definition. In this work, it is indicated how Factorization Machines (FM) can be utilized as a nonexclusive way to deal with tackling the two tracks. FMs consolidate the adaptability of highlight building with the bit of leeway's factorization models. This paper in the blink of an eye presented FMs and presents for the two undertakings in detail the learning destinations and how highlights can be created. For track 1, Bayesian deduction with Markov Chain Monte Carlo (MCMC) is utilized, while, for track 2, stochastic slope plunge (SGD) and MCMC based arrangements are consolidated.

This work [5] investigates a model-based methodology for a proposal in informal communities, utilizing a grid factorization procedure. Progressing past work, fuse the component of trust proliferation into the model. Trust proliferation is an essential marvel in the sociologies, in interpersonal organization examination, and trust-based proposal. Directed investigations on two genuine informational indexes, the open area Epinions.com datasets, and a lot bigger dataset and a lot bigger dataset that has as of late crept from Fluster. Their trials exhibit that displaying trust proliferation prompts a considerable increment in proposal precision, specifically for cold-start clients. 
Cold-start models [6] are special cases of a linear content-based model, with the implicit constraint on the weights. Utilizing this knowledge, this paper proposed LoCo (Low-rank direct cold start), another model for a cool beginning suggestion dependent on three fixings: (a) straight relapse to become familiar with an ideal weighting of social signs for inclinations (b) a low-rank parameterization social signs for inclinations, of the loads to conquer the high dimensionality regular in social information, (c) versatile learning of such low-position loads utilizing randomized SVD (Singular Value Decomposition) probes four genuine world datasets show that LoCo yields huge upgrades over cutting edge cold-start recommenders that misuse high-dimensional interpersonal organization metadata.

This is work [7] proposes a nonexclusive Attributed Social Network Embedding system (ASNE), which learns portrayals for social on-screen characters (i.e., hubs) by saving both the auxiliary nearness and characteristic vicinity while the basic closeness catches the worldwide system structure, the quality vicinity represents the homophile impact. To legitimize their proposition, the lead broad examinations on four true interpersonal organizations. Contrasted with the best in class organize inserting approaches, ASNE can learn increasingly instructive portrayals, accomplishing significant gains on the errands of connection forecast and hub grouping. In particular, ASNE altogether outflanks node2vec with a 8.2 percent relative enhancement for the connection expectation task, and a 12.7 percent gain on the hub grouping task.

This is work [8] proposes a novel regularity based shared separating suggestion strategy named Tyco (Typicality based collaborative filtering approach). An unmistakable component of ordinariness based $\mathrm{CF}$ (Collaborative Filtering) is that it discovers "neighbors" of client's dependent on client averageness degrees in client gatherings (rather than the curated things of clients, or normal clients of things, as in conventional $\mathrm{CF}$ ). To the extent, we might know, there has been no earlier work on examining $\mathrm{CF}$ suggestion by joining object averageness. Tyco beats numerous $\mathrm{CF}$ proposal techniques on suggestion precision (regarding MAE) with an improvement of in any event 6.35 percent in Movie lens informational collection, particularly with inadequate preparing information ( 9.89 percent enhancement for MAE) and has lower time cost than other $\mathrm{CF}$ strategies. Further, it can get progressively precise expectations with fewer enormous mistake forecasts.

\section{DESIRED PROPERTIES OF RECOMMENDATION}

1.Accuracy of relevant recommendation: A proposal is significant in case it interests the customer. The precision of relevant recommendations is the extent of the quantity of huge proposition to the amount of all things considered recommendations. Different estimations for evaluating accuracy can be found in. This limit can be used to review the reasonability similarly to the estimation of the system. While learning the customer's profile, if less significant test recommendations are made, the structure's precision rate may fall. Courses of action need to ensure by and large accuracy. One way to deal with do this is to pick a negligible number of inquiries things with the best guidance.

2.Reducing Bias: Ratings are relied upon to catch associations among clients and things. Be that as it may, a few appraisals are given freely of collaborations. For example, famous things will in general get high evaluations, and a few clients rate things regardless of their involvement with utilizing the thing. Such one-sided evaluations hamper the personalization of suggestions. Arrangements use gauge indicators of predisposition elements to lessen the inclination, yet they require the rating history for precisely displaying them. For the chilly beginning issue, reasonable techniques should exist to catch the rating anomalies from the beginning.

3.Adaptability: It is appropriate that answers are versatile with acknowledgment to the working guideline of the proposed framework there are one of a kind sifting procedures and different rating design that can be used by an RS. A versatile arrangement lessens the work required to make it good with the framework.

4.Diversity: Users have a tendency to be extra satisfied with guidelines while there's a higher intra-listing diversity

e.g. objects from one-of-a-kind artists.

In the recommendation of e-commerce information collection, the phase is very important, that phase is going through the two different ways-

1.Explicit - Explicit is a specific data collection system is simple but difficult to work with. 
Assumedly, the evaluations that a shopper gives might be immediately deciphered as the individual's other options, making it simpler to make extrapolations from realities to are expecting fate scores.

2.Implicit - Implicit information is easy to accumulate in large amounts with no more noteworthy endeavor with respect to the individual. Lamentably, it is a terrible part progressively hard to work with [10]. The point is to change an individual to lead into client prospects, yet it brings for getting more than one hindrance.

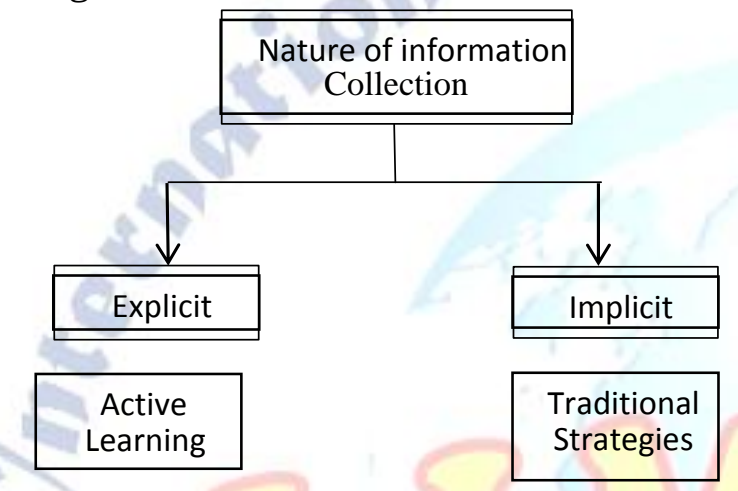

Fig. 1: Nature of information collection

A. Active Learning: Requesting that the client's rate a few things is one method of acquiring data expressly. In any case, rather than choosing arbitrary things, it is alluring to choose important things, which would help in improving the framework's information on the client's inclinations. Simultaneously, the number of choices things must be little so as not to overpower the client. Dynamic Learning (AL) is a valuable strategy for choosing a lot of things that are insignificant and important [11].

\section{B. Adapting Traditional Filtering Strategies:} Conventional sifting procedures, for example, content separating have constrained degree neglected beginning situation because of a deficiency of data. For instance, the area based investigation client rating history to decide an area for them. One approach to adjust them to the virus start situation is to change them to work with inadequate information. In, another likeness degree for an area empowers the suggestion framework to decide an area for another client. The proposed comparability measure is a weighted direct blend of more straightforward similitude gauges that are improved utilizing a neural system.

\section{PROPOSED SYSTEM ARCHITECTURE}

This proposed framework bunched the things which will best match to client profile likewise client's post. The following is a mix of the social and internet business webpage. This framework gives more exactness for separating the two developments. At the present time, he can use the two destinations in a similar territory if any client can buy any item from an online business site; he can a send survey of the item on his/her social website. When a client sends that audit then that post is refreshed on social site for item suggestion to his/her companions. In this framework, going to make two sites, to be specific social destinations and web-based business locales. The number of clients are associated with the two destinations. A Social site has capacities like Create a profile, Update profile, sending companion demands, giving input, sharing the item data. The E-trade site additionally has highlights like Check item, Buy the item, Feedback, Ranking the item. Mining the results from the two areas clients can wind up more noteworthy close with appropriate item proposals and sell on internet business furthermore get extended by getting analysis from clients.

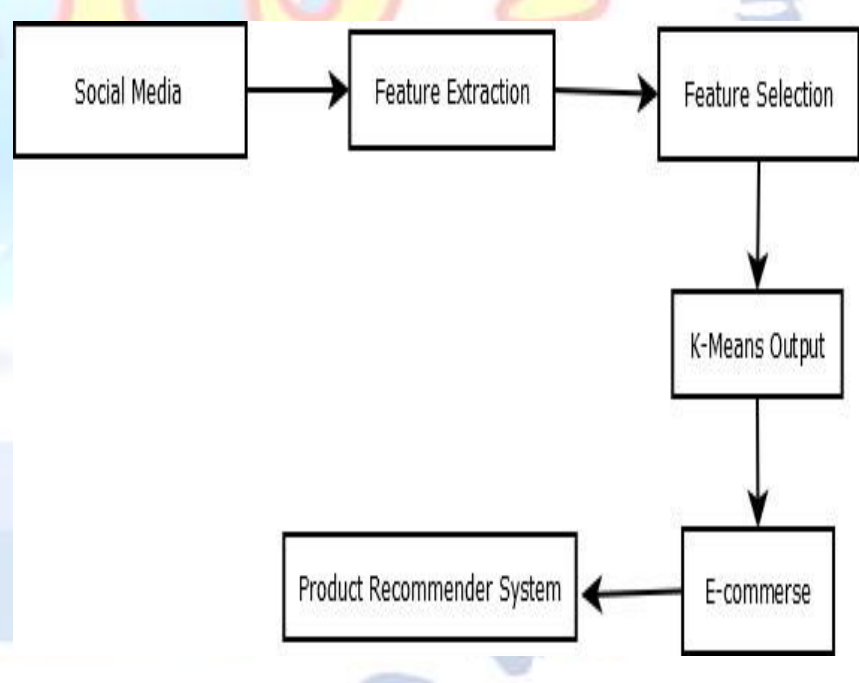

Fig. 2. System Architecture

\section{CONCLUSION}

In this framework, mostly considered a novel issue, cross-site cold-start item proposal, i.e., suggesting items from client post and client profile. In this framework, the fundamental thought of the online business sites, clients, and items can be spoken to in the equivalent inactive component space through element learning with the repetitive neural systems and k-implies bunching calculation. Using a lot of associated customers 
across both electronic business destinations and long-extend casual correspondence regions as a framework, figure out how to incorporate mapping limits using an adjusted slant boosting trees, which maps client's properties removed from person to person communication locales onto highlight portrayals gained from internet business sites. All answers for the new client cold-start issue gather missing data about clients. As indicated by the writing study, the current arrangements are partitioned into two classes depending on how they gather the missing data unequivocally or certainly. The methods are looked at, and their qualities and restrictions have been distinguished.

\section{REFERENCES}

[1] Wayne Xin Zhao, Sui Li, Yulan He, Edward Y. Chang, JiRong Wen and Xi-aoming Li, "Connecting Social Media to Ecommerce: Cold-Start Product Recommendation using Microblogging Information", in IEEE (Institute ofElectrical and Electronics Engineers), 2015.

[2] J. Wang, W. X. Zhao, Y. He, and X. Li, "Leveraging product adopter in-formation from online reviews for product recommendation", in ICWSM (International Conference on Weblogs and Social Media), 2015.

[3] M. Zhang, J. Tang, X. Zhang, and X. Xue, "Addressing cold start in recommender systems: a semi-supervised co-training algorithm", in SIGIR (Special Interest Group on Information Retrieval), 2014.

[4] J. Lin, K. Sugiyama, M. Kan, and T. Chua, "Addressing cold start in app recommendation: latent user models constructed from twitter followers", in SIGIR (Special Interest Group on Information Retrieval), 2013.

[5] Steffen Rendle, "Social Network and Click-through Prediction with Factorization Machines Social Network Analysis" University of Konstanz 78457 Konstanz, Germany, 2012.

[6] S. Sedhain A.K. Menon, S. Sanner, L. Xie and D. Braziunas $\mathrm{S}$, "Low-rank linear cold-start Recommendation from social data", in AAAI (Association for the Advancement of Artificial Intelligence), 2017

[7] L. Liao, X. He, H. Zhang, and T. -S, Chua, "Attributed Social network embedding," IEEE TKDE (Institute ofElectrical and Electronics EngineersTransactions on Knowledge and Data Engineering), vol. 30, no. 12, pp. 2257-2270, 2018

[8] Y. Cai, H. -f. Leung, Q. Li, H. Min, J. Li "Typically-based Collaborative filtering recommendation," IEEE TKDE (Institute ofElectrical and Electronics EngineersTransactions on Knowledge and Data Engineering), vol. 26, no. 3, pp. 766-779, 2014

[9] Vimala. Vellaichamy, Vivekanandan. Kalimuthu, "Hybrid Collaborative Movie Recommender System Using Clustering and Bat Optimization" ISSN (International Journal of Intelligent Engineering and Systems),doi: $10.2478 /$ slgr-2014

[10] Jayawardhena, L. T. Wright, and C. Dennis, "Consumers online: intentions, orientations and segmentation" International Journal of Retail \& Distribution Management, 35:515526, 2007.

[11] J. L. Herlocker, J. A. Konstan, and J. Riedl, "Explaining collaborative filtering recommendations", In Proceedings of the 2000 ACM conference on Computer supported cooperative work, pages 241250. ACM(Association for Computing Machinery) 2000.

[12] Gope, J., \& Jain, S. K. (2017). A survey on solving cold start problem in recommender systems. 2017 International Conference on Computing, Communication and Automation (ICCCA). doi:10.1109/ccaa.2017.8229786

[13] L. Shi, W. X. Zhao and Y. D. Shen, "Local representative-based matrix Factorization for Cold-Start Recommendation, "ACM TOIS (Association for Computing Machinery Transactions on Information Systems) Vol. 36, no.2, p.27.2017.
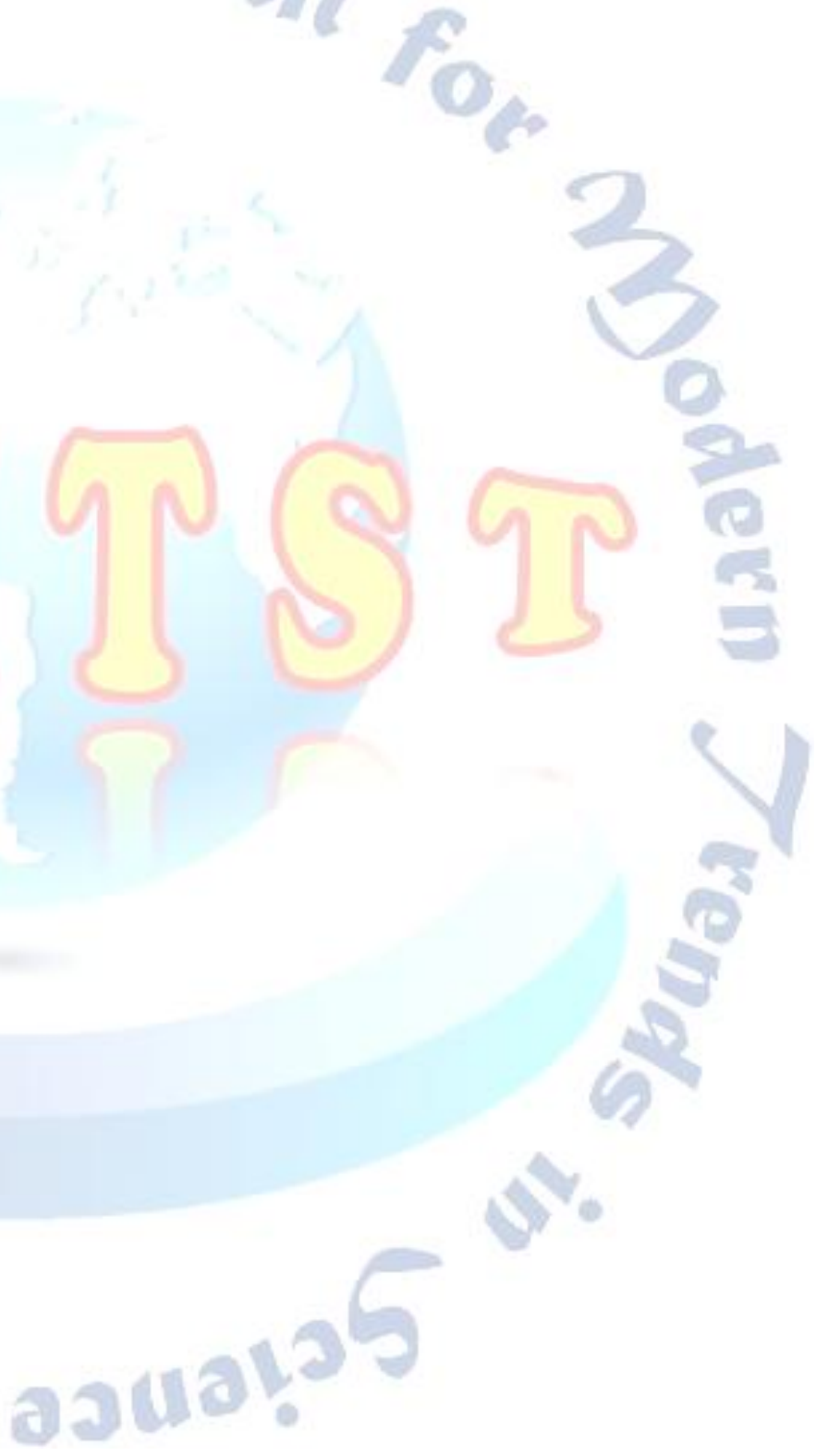